\section{International Scientific Journal Theoretical \& Applied Science}

p-ISSN: 2308-4944 (print) e-ISSN: 2409-0085 (online)

Year: $2018 \quad$ Issue: $01 \quad$ Volume: 57

Published: $30.01 .2018 \quad$ http://T-Science.org
Guljan Risbayevna Dyusebayeva Lecturer of Propedeutics and Internal disease department International Kazakh-Turkish University by name Yassavi, Kazakhstan gulzhan.gulja86@mail.ru

Sarviniso Islamovna Ibragimova Master of medical science, lecturer of Propedeutics and Internal disease department International Kazakh-Turkish University by name Yassavi, Kazakhstan sarvinsa@mail.ru

\title{
TRADITIONAL RISK FACTORS OF CARDIOVASCULAR DISEASES. (LITERATURE REVIEW)
}

\author{
Abstract: The article presents a review of literature data of the prevalence and frequency traditional risk \\ factors occurrence of cardiovascular diseases. Risk factors are considered depending on gender, age and \\ nationality. \\ Key words: cardiovascular diseases, risk factors, gender, age, nationality \\ Language: Russian \\ Citation: Dyusebayeva GR, Ibragimova SI (2018) TRADITIONAL RISK FACTORS OF \\ CARDIOVASCULAR DISEASES. \\ LITERATURE REVIEW. ISJ Theoretical \& Applied Science, 01 (57): 156-160. \\ Soi: http://s-o-i.org/1.1/TAS-01-57-28 Doi: crossef https://dx.doi.org/10.15863/TAS.2018.01.57.28

\section{УДК 616.13.}

\section{ТРАДИЦИОННЫЕ ФАКТОРЫ РИСКА СЕРДЕЧНО-СОСУДИСТЫХ ЗАБОЛЕВАНИЙ. (ЛИТЕРАТУРНЫЙ ОБЗОР)}

Аннотация: В статье представлен обзор литературных данных по изучению распространенности и особенности частоты встречаемости традиционных факторов риска сердечно-сосудистых заболеваний. Факторы риска рассматриваются в зависимости от пола, возраста и наџиональности.

Ключевые слова: сердечно-сосудистые заболевания, факторы риска, пол, возраст, начиональность

\section{Введение.}

Сердечно-сосудистые заболевания (СС3) представляют большую социальноэкономическую проблему, так как занимают ведущее место в структуре заболеваемости и утраты трудоспособности не только в экономически развитых, но и в развивающихся странах [1].

По данным центров, контролирующих заболеваемость и их профилактику (Centers for Disease Control and Prevention), средняя продолжительность жизни была бы на 10 лет больше при отсутствии столь высокой распространенности СС3, охватывающих все страны и континенты [2].

Ежегодно от СС3 в мире умирают около 16,7 млн человек [3]. В структуре смертности от всех причин СС3 составляют около 30\% (27,1\% у мужчин и $31,7 \%$ у женщин). В экономически развитых странах последние три десятилетия отмечается отчетливая тенденция к снижению доли СС3 в структуре общей смертности, их доля не превышает $40 \%$, и только 1 из 5 смертей случается в возрасте до 70 лет. В развивающихся странах доля смертей от ССЗ продолжает расти, и почти каждая вторая смерть от СС3 наступает в возрасте до 70 лет. Причина такого явления в том, что в развитом экономически стабильном обществе удается получить хороший эффект от научно обоснованных программ борьбы с CC3 $[4,5]$.

\section{Основная часть.}

В развитии СС3, в частности ишемической болезни сердца (ИБС) играют большую роль факторы риска: модифицируемые (повышенное артериальное давление (АД), курение, дислипидемия, сахарный диабет, ожирение, диетические факторы, низкая физическая активность, тромбогенные факторы, чрезмерное 
употребление алкоголя) и немодифицируемые (индивидуальный семейный анамнез СC3, возраст, пол) [6]. В исследовании Kulkayeva G, Harun-Or-Rashid M, Yoshida Y, Tulebayev K, Sakamoto J. приводится, что для казахов наиболее характерными факторами риска являются табакокурение, избыточный вес, артериальная гипертензия (АГ) и употребление алкоголя, при этом только $25 \%$ исследуемых из 611 имели хорошие знания о факторах риска СС3 [7].

Влияние сахарного (СД) на развитие и течение ИБС активно изучалось в таких странах как США, Англия, Австралия, Китай подтверждая, что СД является фактором риска развития ИБС в дальнейшем [8,9]. Одним из крупних исследований в этой области является когортное исследование The Framingham Study продолжавшееся 25 лет. В проспективную когорту вошли 5209 мужчин и женщин, которые на момент обследования были в возрасте 30-62 лет. Все участники обследовались раз в два года для определения динамики СС3. В ходе исследования связь прервалась с $3 \%$ участниками. По результатам данного исследования за 20-летний период наблюдения отмечается, что распространенность СД увеличивается с возрастом, составляя у мужчин 7,8\%, среди женщин 6,2\%. Заболеваемость СС3 была выше среди больных страдающих СД, чем в контрольной группе (без СД). Частота развитие ИБС среди мужчин с СД была два раза выше, а среди женщин 3 раза выше чем в контрольной группе. Хотя данный анализ проведен на ограниченное количество больных с СД, в ходе данного исследования получена значимая ассоциация СД и СС3 [10]. Аналогичные результаты получены в исследовании проведенной в Австралии [11]. Интересные результаты получены в исследовании проведенное Simons LA, Friedlander Y, McCallum J, Simons J, которое исследует возникновение ИБС у лиц с верхней границей нормы уровня глюкозы натощак. Согласно данному исследованию заболеваемость ИБС в течение 113 месяцев статистически значимо была выше только у женщин. При этом развитие инсульта не была связано с уровнем глюкозы натощак [12]. В метаанализе проведенном в Калифорнийском университете Соединенных Штатов Америки получены противоположные результаты. По заключению данного метаанализа после поправки на возраст и факторы риска ИБС никакой связи ИБС у женщин страдающих СД не обнаруживалась, а у мужчин эта связь оставалась значимым даже после корректировки на все факторы риска ИБС. Таким образом, связь ИБС между мужчинами и женщинами страдающие СД до сих пор остается противоречивыми [13].
СС3 часто приводят к развитию тяжелых осложнений вплоть до инвалидности. В Framingham Heart Study была рассмотрена связь между инвалидностью и СС3 27 лет после первоначального осмотра 2021 участников (между 1976 и 1978 г.), пациенты были опрошены c целью определения их самооценки, способности выполнять различную физическую деятельность. Среди женщин гипертония, высокий индекс массы тела а также сахарный диабет, а среди мужчин гипертония была связана с инвалидностью. Исследование показало, что уровень холестерина не связан с инвалидностью. Таким образом, авторы считают, что некоторые факторы риска СС3 должны быть рассмотрены в рамках усиленной настороженности для предупреждения дальнейшего развития инвалидности [14]. В данном исследовании также рассматривалась инвалидность вследствие инсульта среди 2540 мужчин и женщин. Установлены, что у $12 \%$ мужчин, и только $3 \%$ женщин наблюдается инвалидность вследствие инсульта [15].

Этнические различия в отношении факторов риска СС3 исследованы в Китай, Сыньцзяне среди казахов, уйгуров, монголов и представителей Ханского народа, которые являются 4-мя крупными этническими группами проживающие в Сыньцзяне. Данное кросссекционное исследование проводилось с 2002 по 2008 годы среди 4421 казахов, 3884 представители Ханского народа, 3218 уйгуров, 892 монголов в возрасте старше 30 лет. С помощью анкетирования определили демографические особенности, наличия инфаркта миокарда, инсульта, сердечной недостаточности, АГ, лечение этих заболеваний, уровень холестерина, СД, наследственная отягощенность, избыточный вес, употребление алкоголя, курение в анамнезе. Все данные были собраны в местной поликлинике за исключением небольшого количества участников которые были обследованы на дому. АД измерено по протоколу Американской сердечной ассоциации, согласно которому за АГ принимались значения САД $\geq 140$, диастолического (ДАД) $\geq 90$. Рост и масса тела определены по стандартному протоколу, за избыточную массу тела принимались значения индекса массы тела $\geq 24$. Из лабораторных исследований были определены: общий холестерин, холестерин липопротеидов высокой плотности (ЛПВП), холестерин липопротеидов низкой плотности (ЛПНП), триглицериды (ТГ), глюкоза крови натощак. За дислипидемию принимались следующие значения: холестерин $\geq 5,18$ ммоль/л, триглицериды $\geq 1,7$ ммоль/л, холестерин ЛПВП $<1,04$ ммоль/л, холестерин ЛПНП $\geq 3.37$ ммоль / л. За СД принимали уровень глюкозы в плазме крови натощак 
27.0ммоль/л, а также СД в анамнезе. При статистической обработке распределение факторов риска анализировалось с помощью однофакторного дисперсионного анализа, различия между группами сравнивали с помощью хи-квадрат. Анализ факторов риска СC3 был определен с помощью логистической регресии. По результатам данного исследования САД, ДАД, а также уровни ТГ и холестерин ЛПНП были значительно выше у казахов и монгольского населения чем для уйгуров и представителей Ханского народа (P <0,001). ИМТ, распространенность дислипидемии и избыточный вес также оказались значительно выше для казахского, уйгурского и монгольского населения, чем для Ханского населения (p $<0,001)$. Уровни ТГ были значительно выше у уйгуров и населения Хань, чем для казахов и монгольского населения $(\mathrm{P}<0,001)$. Уровень холестерина ЛПВП оказался значительно ниже, для населения уйгуров по сравнению с казахами, монголами и Ханского населения (Р <0,001). Распространенность употребления алкоголя и курение были значительно ниже у уйгуров и казахов, чем у монголов и Ханского населения (p $<0,001)$. Распространенность СД была значительно выше, для уйгуров чем у Ханского, казахского и монгольского населения $(\mathrm{P}<0,001)$. Таким образом, результаты настоящего исследования показали, что 90,4\% казахов, 91,9\% уйгуров 90,4\% монголов 85,1\% населения Хан имели по крайней мере один из следующих факторов риска СС3: артериальная гипертензия, дислипидемии, СД, курение, избыточный вес. Исходя из этого, исследователи прелагают учитывать этноспецифические особенности при разработке профилактических мероприятий [16].

Влияние АГ на кардиоваскулярную заболеваемость и смертность является объектом пристального изучения во всем мире. АГ относится к проблемам, которые представляют собой фундаментальную основу для сердечнососудистого континуума, и обусловливает целый ряд причинно-следственных взаимосвязей, которые неблагоприятно сказываются на риске развития различных заболеваний и осложнений. АГ и ассоциированные с ней патологические нарушения тяжелым бременем ложатся на общество - как в силу высокой распространенности в популяции, так и из-за тяжелых последствий [17]. Хотя высокое АД, уровень холестерина избыточная масса тела исторически считались риски как "Западными", их влияние в настоящее время признается в качестве глобального: они уже являются причиной заболеваемости СС3 в странах со средним и низким уровнем дохода [18].

Масштабные эпидемиологические и клинические исследования неоднократно демонстрировали выраженное неблагоприятное влияние повышенного АД на риск кардиоваскулярных событий [19,20]. В 2008 г. были опубликованы данные Международного общества гипертензии (International Society of Hypertension) в котором оценивалось бремя АГ в мире в 2001 г. [21]. Авторы подтвердили, что в популяции людей 30 лет и старше систолическое АД $\geq 115$ мм рт. ст. ассоциировано с 7,6 млн случаев преждевременной смерти во всем мире (что составляет $13,5 \%$ от общего количества умерших) и 92 млн (6\% от общего количества) утраченных лет здоровой жизни, или индекса DALY (disability-adjusted life years - комплексный показатель, который учитывает потери здоровой жизни в результате преждевременной смерти и утраты здоровых лет жизни по причине временной или постоянной нетрудоспособности). Кроме того, такие уровни АД были причиной $54 \%$ всех инсультов, 47\% всех случаев ИБС и $25 \%$ остальных СС3. При этом не было обнаружено убедительных доказательств того, что АД ниже 115/75 мм рт. ст. неблагоприятно влияет на сердечно-сосудистый риск [22].

Одно из крупных исследований по изучению АГ в странах бывшего Советского Союза проведено совместно с Лондонской школой гигиены и тропической медицины и Центра социологических исследований Московского униветситета с 2001 по 2010 годы. По мнению авторов, страны бывшего Советского Союза пострадали в отношении психического здоровья, особенно в период распада СССР. Для изучения влияния стресса на развитие АГ были вклячены 9 стран бывшего Советского Союза: Армения, Беларуссия, Грузия, Кыргызстан, Молдова, Россия, Украина и Казахстан. Выборка состояла из 18424 человек в 2001 году, и 18000 человек в 2010 году с большей долей женщин (57\% женщин, 43\% мужчин). По результатам данного исследования отмечается некоторое уменьшение уровня психического стресса в 2010 году по сравнению с 2001 годом, которое объясняется улучшением экономического состояния и адаптацией на политическую перестройку. Тем не менее, уровень психического стресса значимо была связана с развитием АГ особенно у женщин. Как предполагают авторы, это связано тем, что в странах бывшего СССР придается меньше значений ментальным расстройствам, а также роль стресса в развитии заболеваний. Таким образом, 9,9\% участников с АГ в 2010 году имели высокий уровень стресса, чем в общей популяции по сравнению с 4,9\% 2001 году. Участники с АГ в 5 раз чаще испытывали стресс, чем не имеющие данного заболевания в Армении и Кыргызстана, но для Казахстана эти результаты не были статистически значимы. Установлены основные предраспологающие 
факторы развития АГ, такие как женский пол, возраст старше 50 лет, низкий уровень образования и экономической обеспеченности, а также ограниченный доступ к медицинским услугам и лекарствам [23].

Интересно, что АГ и ассоциированные с ней проблемы вносят значительный вклад в заболеваемость даже в тех странах, которые традиционно являются центром других тяжелых проблем, обусловливающих раннюю смерть значительной части населения, - многолетних войн, голода, изнуряющих эпидемий инфекционных болезней, в том числе ВИЧ/СПИДа. Например, в некоторых регионах Африки, несмотря на массу других болезней, не актуальных для развитых стран мира, широко распространенным заболеванием среди взрослых является АГ, которая связана с социоэкономическим стрессом [24]. Противоположно этому в другом исследовании проведенном на территории Африки не нашли никакой связи между стрессом и развитием АГ [25].

В исследовании, проведенном C.M. Lawes, c объемом выборки 660000 человек, изучались гендерные различия в развитии АГ. В ходе этого исследования обнаружилось, что в среднем АД немного ниже среди популяции взрослых женщин, чем среди популяции взрослых. Однако если оценить уровни АД у лиц разного возраста, оказывается, что у женщин 30-44 лет АД в среднем ниже, чем у мужчин той же возрастной группы, но после наступления климакса АД у женщин достаточно быстро и значительно увеличивается, и в возрасте $\geq 60$ лет оно в среднем уже выше у женщин, чем у мужчин [26]. Аналогичные результаты были получены и в ряде других исследований $[27,28]$.

Распространенность АГ и связанных с ней заболеваний, и применение современных антигипертензивных препаратов существенно отличаются в развитых странах мира (США, Канаде, Австралии, странах Европы) и в странах с низким уровнем дохода на душу населения. В то время как в развитых странах в последние годы отмечается заметный прогресс в лечении и профилактике АГ, инсультов, инфарктов и других кардиоваскулярных заболеваний, ситуация в развивающихся государствах остается неблагоприятной, а зачастую даже ухудшается. АГ является главным фактором риска сердечнососудистой заболеваемости. Наличие АГ повышает риск развития ИБС, в том числе острого инфаркта миокарда и внезапной коронарной смерти более чем в 2 раза, а возникновение сердечной недостаточности и инсульта - более чем в 3 раза [29].

\section{Выводы.}

Таким образом, изучению факторов риска CC3 посвящены многие работы как зарубежных, так и отечественных исследователей. Существуют множество противоречивых выводов проведенных исследований, что требует проведение дальнейших работ для улучшения профилактических мер столь распространенных CC3.

\section{References:}

1. Shcherbakova E. (2011) V 2001-2009 godakh ot 56 do $58 \%$ smertey v SNG byli vyzvany boleznyami sistemy krovoobrashcheniya, ot 12 do $13 \%$-zlokachestvennymi novoobrazovaniyami // Demeskop Weekly. 2011.-№469-470.

2. (2018) Available: http://who.int/mediacentre/factsheets/fs317/ru/ (Accessed: 10.01.2018).

3. (2002) Doklad $« \mathrm{O}$ sostoyanii zdravookhraneniya v Evrope, 2002 g.» - pressreliz ERB VOZ/15/02, Kopengagen, 17 sentyabrya $2002 \mathrm{~g}$.

4. Bury M. (1994) Helth promotion and lay epidemiology:a sociological view // Health Care Analysis. - 1994. - Vol. 2. - p. 23-30.
5. Sans S., Kesteloot H., Kromhout D. (1997) The burden of cardiovascular diseases mortality in Europe. Task Force of the European Society of Cardiology on Cardiovascular Mortality and Morbidity Statistics in Europe // Eur. Heart J. 1997. - Vol. 18. - p. 1231-1248.

6. O.A.Kislyak, N.V.Malysheva, N.N.Chirkova (2017) Klinicheskaya gerontologiya// Faktory riska serdechno-sosudistykh zabolevaniy $\mathrm{V}$ razvitii bolezney svyazannykh s aterosklerozom//..3-str.

7. Kulkayeva G, Harun-Or-Rashid M, Yoshida Y, Tulebayev K, Sakamoto J. (2012) Nagoya J Med Sci. 2012. Cardiovascular disease risk factors among rural Kazakh population. 


\begin{tabular}{l|lr|ll|ll} 
& ISRA (India) & $=\mathbf{1 . 3 4 4}$ & SIS (USA) & $=\mathbf{0 . 9 1 2}$ & ICV (Poland) & $=\mathbf{6 . 6 3 0}$ \\
Impact Factor: & ISI (Dubai, UAE) $=\mathbf{0 . 8 2 9}$ & PUHЦ (Russia) $=\mathbf{0 . 2 0 7}$ & PIF (India) & $=\mathbf{1 . 9 4 0}$ \\
& GIF (Australia) & $\mathbf{0 . 5 6 4}$ & ESJI (KZ) & $=4.102$ & IBI (India) & $=\mathbf{4 . 2 6 0}$ \\
& JIF & $=\mathbf{1 . 5 0 0}$ & SJIF (Morocco) & $=\mathbf{2 . 0 3 1}$ & & \\
\hline
\end{tabular}

8. Kanaya AM, Grady D, Barrett-Connor E. (2002) Arch Intern Med. Explaining the sex difference in coronary heart disease mortality among patients with type 2 diabetes mellitus: a meta-analysis.

9. Bulugahapitiya U, Siyambalapitiya S. (2009) Diabet Med. Is diabetes a coronary risk equivalent? Systematic review and metaanalysis.

10. Kannel WB, McGee DL (1979) Circulation. Diabetes and cardiovascular risk factors: the Framingham study.

11. Simons LA, McCallum J. (2017) Aust N Z J Med. Diabetes, mortality and coronary heart disease in the prospective Dubbo study of Australian elderly.

12. Kanaya AM, Grady D, Barrett-Connor E. (2002) Arch Intern Med.. Explaining the sex difference in coronary heart disease mortality among patients with type 2 diabetes mellitus: a meta-analysis.

13. Simons LA, Friedlander Y. (2000) Aust N Z J Med.. Fasting plasma glucose in non-diabetic elderly women predicts increased all-causes mortality and coronary heart disease risk.

14. Pinsky JL, Branch LG, Jette AM, Haynes SG, Feinleib M, Cornoni-Huntley JC, Bailey KR. (1985) Am J Epidemiol.. Framingham Disability Study: relationship of disability to cardiovascular risk factors among persons free of diagnosed cardiovascular disease.

15. Jette AM, Pinsky JL, Branch LG. (1988) J Clin Epidemiol.. The Framingham Disability Study: physical disability among community-dwelling survivors of stroke.

16. Nanfang Li, Hongmei Wang, Zhitao Yan, Xiaoguang Yao, Jing Hong and Ling Zhou (2012) BMC Public Health. Ethnic disparities in the clustering of risk factors for cardiovascular disease among the Kazakh, Uygur, Mongolian and Han populations of Xinjiang: a cross-sectional study.

17. (2018)

Available: http://medreview.com.ua/issues/9/article1/ (Accessed: 10.01.2018).

18. Arnold M, Pandeya N. (2015) Lancet Oncol. Jan. Global burden of cancer attributable to high body-mass index in 2012: a populationbased study.

19. Collins R, Peto R, MacMahon S. (1990) Lancet.. Blood pressure, stroke, and coronary heart disease. Part 2, Short-term reductions in blood pressure: overview of randomised drug trials in their epidemiological context.

20. Lawes CM, Bennett DA, Lewington S, Rodgers A. (2002) Semin Vasc Med.. Blood pressure and coronary heart disease: a review of the evidence.

21. Lawes C.M., Vander Hoorn S., Rodgers A. (2001) International Society of Hypertension. Global burden of blood-pressure-related disease, 2001. Lancet 2008.

22. (2009) Medicine Review 2009; 4 (09): 6-11

23. Footman K, Roberts B, Tumanov S, McKee M. (2013) J Public Health (Oxf).. The comorbidity of hypertension and psychological distress: a study of nine countries in the former Soviet Union.

24. Opie LH, Seedat YK. (2005) Circulation.. Hypertension in sub-Saharan African populations.

25. Agyei B, Nicolaou M, Boateng L, Dijkshoorn H. (2014) BMC Public Health.. Relationship between psychosocial stress and hypertension among Ghanaians in Amsterdam, the Netherlands--the GHAIA study.

26. Lawes CM, Vander Hoorn S, Law MR, Elliott P, MacMahon S. (2006) J Hypertens.. Blood pressure and the global burden of disease 2000. Part 1: estimates of blood pressure levels.

27. Martins D, Nelson K, Pan D, Tareen N, Norris K. (2001) The effect of gender on age-related blood pressure changes and the prevalence of isolated systolic hypertension among older adults: data from NHANES III. J Gend Specif Med. 4(3):10-3, 20.

28. Clara JG, De Macedo ME. (2007) Rev Port Cardiol.. Prevalence of isolated systolic hypertension in the population over 55 years old. Results from a national study.

29. Safargalieva L.Kh. (2013) Izmeneniya na EKG pri apikal'noy gipertrofii levogo zheludochka / Vestnik sovremennoy klinicheskoy meditsiny 2013 Tom 6, vyp. 4. p. 52-53 